\title{
Reduced-Order Modeling for Flexible Spacecraft Deployment and Dynamics
}

\author{
Michael A. Marshall,* and Sergio Pellegrino ${ }^{\dagger}$ \\ California Institute of Technology, Pasadena, California, 91125
}

\begin{abstract}
The present work investigates reduced-order modeling for ultralight, packageable, and self-deployable spacecraft where reduced-order models (ROMs) are required to simulate deployment, structural dynamics during spacecraft maneuvers, and for real-time applications in trajectory optimization and control. In these contexts, ultralight, flexible spacecraft dynamics are characterized by geometrically nonlinear structural deformations combined with large rigid body motions. An approach based on proper orthogonal decomposition (POD), energy-conserving sampling and weighting (ECSW), and a floating frame of reference (FFR) is proposed to construct accurate and efficient ROMs. The proposed approach is then tested on a benchmark problem that involves geometrically nonlinear deformations, large rigid body motions, and strain energy release during dynamic snap-back, the last of which is analogous to the energy release during deployment. The resulting ROM for this benchmark problem is approximately $20 \%$ the size of the original full-order model with no appreciable loss of accuracy.
\end{abstract}

\section{Introduction}

Traditionally, spacecraft have been designed as approximately rigid structures with flexible appendages. However, advances in materials and structures are enabling the development of aggressive new structural architectures for ultralight, packageable, and self-deployable spacecraft for applications including solar sailing [1, 2] and space exploration [3]; communications, power transfer, and sensing [4]; and space solar power [5, 6]. Ultralight spacecraft structures trade structural rigidity in the deployed configuration against packaging efficiency in order to stow large apertures in existing launch vehicles. The increased flexibility introduces new challenges for accurately simulating the dynamics of ultralight, flexible spacecraft.

The present work is motivated by problems in dynamics and control of ultralight, flexible spacecraft where reducedorder models (ROMs) are required to simulate deployment events, structural dynamics during spacecraft maneuvers and over long time scales (e.g. an orbit period), and to facilitate real-time simulations for trajectory optimization and control. These problems are characterized by a combination of potentially large (geometrically nonlinear) deformations, i.e., linear-elastic deformations with nonlinearities due to finite rotations in the underlying deformation kinematics, and large rigid body motions. An added complication is that applications in trajectory optimization and control necessitate a Total Lagrangian description of the dynamics to facilitate rigorous theoretical analyses, e.g. of stability. The Total Lagrangian description explicitly parameterizes the model as a function of the independent degrees of freedom, as opposed to the more common Updated Lagrangian formulations typically implemented in commercial finite element software packages.

To that end, the focus of this paper is on reducing geometrically nonlinear structural models for ultralight, flexible spacecraft. The present work considers the reduced-order modeling of a representative problem, the elastica catapult [7], i.e., a slender rod with a tip mass clamped to a rotating base in a gravitational field. A key feature of this problem is the energy released during snap-back; when the gravitational force induced by the tip mass exceeds the rod's Euler buckling load, the rod dynamically snaps to a new equilibrium configuration. The name "elastica catapult" is due to this snap-back phenomenon, which resembles the motion of a catapult's arm and is analogous to the energy release during dynamic deployment [6, 8-10] and localized folding [11] of ultralight spacecraft structures. As a result, the elastica catapult is a relevant benchmark problem for assessing the efficacy of reduced-order modeling methods for ultralight, flexible spacecraft. A ROM derived via a Galerkin projection of a nonlinear finite element model embedded in a lumped mass, floating frame of reference (FFR) and hyper-reduction [12] of the geometric nonlinearities is shown to accurately approximate the response of the elastica catapult. Proper orthogonal decomposition (POD) [13] is used to compute the

\footnotetext{
*Graduate Research Assistant, Graduate Aerospace Laboratories, 1200 E. California Blvd., Mail Code 105-50. mmarshall@ caltech.edu

$\dagger$ Joyce and Kent Kresa Professor of Aerospace and Civil Engineering, Graduate Aerospace Laboratories, 1200 E. California Blvd., Mail Code 105-50. sergiop@ caltech.edu Fellow AIAA.
} 
reduced basis for the Galerkin projection, and energy-conserving sampling and weighting (ECSW) [14, 15] is used for hyper-reduction. In the sequel, this method is referred to as POD-ECSW. The proposed reduced-order modeling approach is capable of modeling large elastic deformations and nonlinear phenomena like dynamic snap-back in systems with large rigid body motions.

This paper is organized as follows: Sec. [I]describes the proposed reduced-order modeling approach for geometrically nonlinear finite element models. Sec. III] shows how to embed the ROM from Sec. II into a FFR to simulate large rigid body motions. Sec. [V then studies reduced-order modeling of the elastica catapult using the methods from Secs. [I] and III] Finally, Sec. V presents the paper's conclusions.

\section{Reduced-Order Modeling for Geometrically Nonlinear Structures}

This section discusses reduced-order modeling for finite element models of geometrically nonlinear structures, i.e., linear-elastic structures where the nonlinearities are due to finite rotations in the underlying deformation kinematics.

\section{A. Governing Equations}

The governing equations for a geometrically nonlinear finite element model are

$$
\mathbf{M u ̈}+\mathbf{D}(\mathbf{u}) \dot{\mathbf{u}}+\mathbf{F}_{n l}(\mathbf{u})=\mathbf{F}_{\text {ext }}
$$

where $\mathbf{u} \in \mathbb{R}^{n}$ is the vector of generalized coordinates, $\mathbf{M} \in \mathbb{R}^{n \times n}$ is the symmetric positive definite mass matrix, $\mathbf{D}(\mathbf{u}) \in \mathbb{R}^{n \times n}$ is the possibly configuration-dependent symmetric positive semi-definite damping matrix, $\mathbf{F}_{n l} \in \mathbb{R}^{n}$ is the vector of internal elastic forces due to the geometric nonlinearity, $\mathbf{F}_{\text {ext }} \in \mathbb{R}^{n}$ is the vector of external generalized forces, and dot notation denotes differentiation with respect to time. The elastic forces $\mathbf{F}_{n l}(\mathbf{u})$ are derived from the strain energy potential.

Nonlinear static solutions and implicit integration schemes both require the tangent stiffness matrix

$$
\mathbf{K}_{T}=\frac{\partial \mathbf{F}_{n l}}{\partial \mathbf{u}}
$$

The tangent stiffness matrix is typically positive definite, but it becomes positive semi-definite at critical points. Physically, a critical point corresponds to a configuration where the structure is unable to support an increase in the external load; to equilibrate the load, the structure dynamically "snaps" to a new equilibrium configuration. This is responsible for the snap-back behavior exhibited by the elastica catapult (Sec. IV).

\section{B. Reduced-Order Modeling via Galerkin Projection and Proper Orthogonal Decomposition}

In general, nonlinear finite element models [Eq. (11] are computationally expensive to simulate. ROMs that approximate the full-order model (FOM) are intended to ease this computational burden without a significant loss of accuracy. For nonlinear static and implicit dynamic solutions, the dimensional reduction associated with a ROM based on a Galerkin projection of the elastic degrees of freedom onto a low-dimensional basis reduces the computational cost of inverting the tangent matrix at each Newton-Rhapson iteration.

The quality of the ROM is determined by the choice of the basis. Several approaches have been proposed in the literature to develop bases for geometrically nonlinear structural models. These include approaches based on normal vibration modes [16, 17], the Craig-Bampton method [18, 19], nonlinear normal modes [20], modal derivatives [21-25], and path derivatives [26]. Data-driven techniques based on POD have also been successfully used for nonlinear reduced-order modeling in a variety of applications; see [13] for a review of POD in structural analysis. POD is simple, non-intrusive, and provides an efficient basis for large deformation problems. For these reasons, it is the method used to identify a reduced basis in this paper.

POD identifies a basis from a set of displacement responses. It starts by assembling the snapshot matrix

$$
\mathbf{U}=\left[\begin{array}{llll}
\mathbf{u}_{1} & \mathbf{u}_{2} & \ldots & \mathbf{u}_{n_{t}}
\end{array}\right]
$$

where $\mathbf{u}_{i}$ denotes snapshot $i$ and $n_{t}$ is the number of snapshots in the training data set. In general, each snapshot is a generalized displacement vector obtained from a static or dynamic simulation of the FOM under a representative loading condition. The POD basis $\Phi \in \mathbb{R}^{n \times r}$ is then obtained by taking the first $r$ left singular vectors of the singular value decomposition of $\mathbf{U}$ where $r \ll n$ is the dimension of the ROM. 
To reduce the full-order finite element model [Eq. 11]], the generalized coordinate vector $\mathbf{u}$ is approximated by a linear combination of reduced generalized coordinates $\hat{\mathbf{u}} \in \mathbb{R}^{r}$; i.e.,

$$
\mathbf{u} \approx \Phi \hat{\mathbf{u}}
$$

Substituting Eq. (4) into Eq. (1) and using a standard Galerkin projection yields

$$
\hat{\mathbf{M}} \ddot{\hat{\mathbf{u}}}+\hat{\mathbf{D}}(\hat{\mathbf{u}}) \dot{\hat{\mathbf{u}}}+\hat{\mathbf{F}}_{n l}(\hat{\mathbf{u}})=\hat{\mathbf{F}}_{e x t}
$$

where $\hat{\mathbf{M}}=\boldsymbol{\Phi}^{T} \mathbf{M} \boldsymbol{\Phi}, \hat{\mathbf{D}}(\hat{\mathbf{u}})=\boldsymbol{\Phi}^{T} \mathbf{D}(\boldsymbol{\Phi} \hat{\mathbf{u}}) \boldsymbol{\Phi}, \hat{\mathbf{F}}_{n l}(\hat{\mathbf{u}})=\boldsymbol{\Phi}^{T} \mathbf{F}_{n l}(\boldsymbol{\Phi} \hat{\mathbf{u}})$, and $\hat{\mathbf{F}}_{\text {ext }}=\boldsymbol{\Phi}^{T} \mathbf{F}_{\text {ext }}$. Likewise, the reduced tangent stiffness matrix is $\hat{\mathbf{K}}_{T}(\hat{\mathbf{u}})=\boldsymbol{\Phi}^{T} \mathbf{K}_{T}(\boldsymbol{\Phi} \hat{\mathbf{u}}) \boldsymbol{\Phi}$. When $\boldsymbol{\Phi}$ is a POD basis, the resulting ROM is termed a POD-Galerkin ROM. By applying the Galerkin projection directly to the nonlinear finite element model, as opposed to a corresponding first-order state space form, the resulting ROM preserves the Lagrangian structure of the FOM [27].

\section{Hyper-Reduction}

Equation (5) highlights the well-known computational bottleneck associated with the Galerkin approach for geometrically nonlinear finite element models. Specifically, evaluating any of the configuration-dependent terms required to simulate Eq. (5) involves function evaluations that scale with the FOM dimension $n$, as opposed to the ROM dimension $r$. To reduce the computational complexity of simulating Eq. (5), the configuration-dependent terms are often further approximated offline using hyper-reduction [12]. A hyper-reduced ROM is then considered efficient if the function evaluations required to simulate the nonlinear terms approximately scale with the ROM dimension $r$. In the context of finite elements, hyper-reduction aims to reduce the computational complexity of the finite element assembly step. For additional discussion of the computational bottleneck in the Galerkin approach, see e.g. [28].

Various hyper-reduction techniques for nonlinear systems of ordinary differential equations have been proposed in the literature, see e.g. [12, 14, 15, 27,-32]. Of these, the methods proposed in Refs. [12, 28-30] are all known to destroy the underlying Lagrangian structure of the FOM [27], which can lead to, among other things, a loss of stability and/or energy conservation in the ROM [15, 27, 33]. Structure preservation in the ROM is believed to be important for correctly modeling structural behavior in the vicinity of critical points, e.g. during snap-back. As a result, the structure and energy-preserving ECSW method developed specifically for nonlinear finite element models is used for hyper-reduction in this paper. The remainder of this section summarizes this method; for additional discussion, see [14, 15].

ECSW uses a training data set defined by the displacement responses in the snapshot matrix [Eq. (3)] to systematically identify a subset of elements in a finite element mesh, a so-called reduced mesh, that with appropriate weights optimally approximates the training data. It hinges on an element-level approximation of the vector of internal elastic forces $\mathbf{F}_{n l}$ that preserves the virtual work of the elastic forces along the virtual displacements defined by the reduced basis $\boldsymbol{\Phi}$. Computational costs are reduced by evaluating the ROM using the reduced mesh instead of the full mesh.

For a finite element model with $n_{e}$ elements, the reduced internal elastic force vector $\hat{\mathbf{F}}_{n l}$ can be expressed as the following summation over the elements:

$$
\hat{\mathbf{F}}_{n l}(\hat{\mathbf{u}})=\sum_{e=1}^{n_{e}} \boldsymbol{\Phi}^{T} \mathbf{L}_{e}^{T} \mathbf{F}_{n l}^{e}\left(\mathbf{L}_{e} \boldsymbol{\Phi} \hat{\mathbf{u}}\right)
$$

where a subscript or superscript $e$ denotes element $e, \mathbf{L}_{e} \in \mathbb{R}^{n_{c}^{e} \times n}$ is the Boolean locator matrix corresponding to element $e, \mathbf{F}_{n l}^{e}$ is the internal elastic force vector for element $e$, and $n_{c}^{e}$ is the number of nodal coordinates per element. The locator matrix $\mathbf{L}_{e}$ indexes the nodal coordinates $\mathbf{u}_{e}$ of element $e$ from the generalized coordinate vector $\mathbf{u}$; i.e., $\mathbf{u}_{e}=\mathbf{L}_{e} \mathbf{u}$. Note that Eq. (6) is an exact representation of the reduced internal elastic force vector. Using ECSW, the reduced internal elastic force vector is then approximated as

$$
\hat{\mathbf{F}}_{n l}(\hat{\mathbf{u}}) \approx \sum_{e \in \hat{E}} \xi_{e}^{*} \boldsymbol{\Phi}^{T} \mathbf{L}_{e}^{T} \mathbf{F}_{n l}^{e}\left(\mathbf{L}_{e} \boldsymbol{\Phi} \hat{\mathbf{u}}\right)
$$

where $\xi_{e}^{*}$ is the weight of element $e$ and $\hat{E}$ is the subset of the elements that defines the reduced mesh. The vector of weights is denoted $\xi^{*} \in \mathbb{R}^{n_{e}}$, and $\xi_{e}^{*}=0$ for any element excluded from the reduced mesh. Hence, the reduced mesh is defined as [14]

$$
\hat{E}=\left\{e \in\left\{1,2, \ldots, n_{e}\right\}: \xi_{e}^{*} \neq 0\right\}
$$

The ECSW tangent stiffness matrix is evaluated by taking the gradient of Eq. (7). 
To maximize sparsity, the weight vector $\xi^{*}$ is the solution to the following offline optimization problem [14]:

$$
\xi^{*}=\arg \min _{\xi \in \Theta}\|\xi\|_{0}
$$

where $\|\cdot\|_{0}$ is the 0 -norm which counts the number of non-zero entries in its vector argument. The solution $\xi^{*}$ is considered sparse when $\left\|\xi^{*}\right\|_{0} \ll n_{e}$. If $\boldsymbol{\xi}^{*}$ is sparse, then evaluating Eq. (7) is efficient in the sense that the number of required function evaluations scales independently of the FOM dimension $n$. The set of feasible solutions $\Theta$ is then defined by [14]

$$
\Theta=\left\{\boldsymbol{\xi} \in \mathbb{R}^{n_{e}}:\|\mathbf{A} \boldsymbol{\xi}-\mathbf{b}\|_{2} \leq \epsilon|| \mathbf{b} \|_{2}, \boldsymbol{\xi} \geq 0\right\}
$$

where $\|\cdot\|_{2}$ is the Euclidean norm, the matrix $\mathbf{A} \in \mathbb{R}^{r n_{t} \times n_{e}}$ and the vector $\mathbf{b} \in \mathbb{R}^{r n_{t}}$ contain the element-level training data, $n_{t}$ is the size of the training data set, and $\epsilon \in[0,1]$ controls the sparsity of $\boldsymbol{\xi}^{*}$. The matrix $\mathbf{A}$ is

$$
\mathbf{A}=\left[\begin{array}{ccc}
\mathbf{a}_{11} & \ldots & \mathbf{a}_{1 n_{e}} \\
\vdots & \ddots & \vdots \\
\mathbf{a}_{n_{t} 1} & \ldots & \mathbf{a}_{n_{t} n_{e}}
\end{array}\right]
$$

where $\mathbf{a}_{i e}=\boldsymbol{\Phi}^{T} \mathbf{L}_{e}^{T} \mathbf{F}_{n l}^{e} \in \mathbb{R}^{r}$ represents the reduced element elastic force vector for element $e$ evaluated at configuration $i$ in the training data set. Similarly, the vector $\mathbf{b}$ is

$$
\mathbf{b}^{T}=\left[\begin{array}{lll}
\mathbf{b}_{1}^{T} & \ldots & \mathbf{b}_{n_{t}}^{T}
\end{array}\right]
$$

where $\mathbf{b}_{i}=\sum_{e=1}^{n_{e}} \mathbf{a}_{i e} \in \mathbb{R}^{r}$ is the assembled reduced elastic force vector [Eq. [6] evaluated at configuration $i$ in the training data set. When $\epsilon=0$, all of the weights are unity and Eq. (7) is equivalent to Eq. (6); i.e., the reduced mesh is identical to the full mesh. To achieve a sparse solution, Ref. [14] suggests using a value for $\epsilon \in[0.01,0.1]$. Feasible solutions are constrained to be non-negative to guarantee a positive semi-definite strain energy.

Equation (9) defines an NP-hard optimization problem, meaning it is not computationally tractable to solve in practice. As a result, instead of solving Eq. (9) directly, an approximate solution for $\boldsymbol{\xi}^{*}$ is obtained by solving a related sparse non-negative least squares (NNLS) problem using an iterative active set algorithm; for details, see [14].

\section{Floating Frame of Reference Formulation}

This section considers the problem of reduced-order modeling for a geometrically nonlinear structure [Eq. [5] ] undergoing large rigid body motions using a lumped mass FFR. The resulting ROM is referred to as a FFR-ROM. A lumped mass formulation is used because it decouples the kinetic energy from the element formulation used in the finite element discretization of the structure [34].

The FFR describes the reference motion of an elastic body. Elastic deformations are then measured with respect to the FFR. This imposes a natural separation between rigid body and elastic coordinates, which allows the elastic coordinates to be directly expressed as a linear combination of reduced basis vectors. The FFR approach is compatible with most existing geometrically nonlinear finite element formulations.

This section first develops the kinematics of the FFR-ROM (Sec. [III.A). It then derives the kinetic energy (Sec. IIII.B) and strain energy (Sec. IIII.C) to facilitate the application of Lagrange's equations to assemble the system's equations of motion (Sec. [III.D). The formulation herein is planar and neglects rotational inertia, as is commonly done in lumped mass structural dynamics models, but it can be readily generalized to three-dimensions and to account for the effects of rotational inertia (see e.g. [34]). The developments in this section largely parallel Chs. 5-6 in [35].

\section{A. Kinematic Description}

Let $\mathbf{r}_{i} \in \mathbb{R}^{2}$ denote the position in inertial reference frame $\mathcal{I}$ of node $i$ in the finite element discretization of an elastic body. In the FFR formulation, the motion of node $i$ in $\mathcal{I}$ is described as the superposition of the rigid body motion of a body reference frame $\mathcal{B}$ relative to $\mathcal{I}$ and an elastic displacement relative to $\mathcal{B}$. In this paper, $\mathcal{B}$ is a nodal-fixed reference frame [36] taken to coincide with the undeformed configuration of the elastic body. With a nodal-fixed reference frame, the boundary conditions remove redundant (rigid body) degrees of freedom with respect to $\mathcal{B}$ in order to uniquely define the elastic displacement field in $\mathcal{B}$. By doing so, the position of the undeformed structure is fixed relative to the origin 
of $\mathcal{B}$. The nodal displacements are then expressed as a linear combination of the ROM's $r$ reduced coordinates in the FFR, analogous to the approach used in [34] for linear FFR-ROMs.

The position of node $i$ in $\mathcal{I}$ is

$$
\mathbf{r}_{i}=\mathbf{R}+\mathbf{T L}_{i}\left(\overline{\mathbf{u}}_{0}+\overline{\mathbf{u}}\right)
$$

where $\mathbf{R} \in \mathbb{R}^{2}$ is the position of the origin of $\mathcal{B}$ in $\mathcal{I}, \mathbf{T} \in \mathbb{R}^{2 \times 2}$ is the reference frame transformation $\mathcal{B} \rightarrow \mathcal{I}$ parameterized by the rotation angle $\psi, \mathbf{L}_{i} \in \mathbb{R}^{2 \times\left(n+n_{f}\right)}$ is the Boolean locator matrix that indexes the translational nodal coordinates, $\overline{\mathbf{u}}_{0} \in \mathbb{R}^{n+n_{f}}$ is the vector of nodal coordinates in the undeformed configuration, $\overline{\mathbf{u}} \in \mathbb{R}^{n+n_{f}}$ is the vector of nodal displacements, $n$ is the number of independent elastic degrees of freedom, i.e., the degrees of freedom not fixed to $\mathcal{B}$, and $n_{f}$ is the number of degrees of freedom fixed to $\mathcal{B}$. The reference frame transformation matrix $\mathbf{T}$ is given by

$$
\mathbf{T}(\psi)=\left[\begin{array}{cc}
\cos (\psi) & -\sin (\psi) \\
\sin (\psi) & \cos (\psi)
\end{array}\right]
$$

with time derivative $\dot{\mathbf{T}}=\mathbf{T}_{\psi} \dot{\psi}$ where $\mathbf{T}_{\psi}=\partial \mathbf{T} / \partial \psi$. Note that both $\mathbf{T}$ and $\mathbf{T}_{\psi}$ are orthogonal; i.e., $\mathbf{T}^{T} \mathbf{T}=\mathbf{I}_{2}$ and $\mathbf{T}_{\psi}^{T} \mathbf{T}_{\psi}=\mathbf{I}_{2}$ where $\mathbf{I}_{2} \in \mathbb{R}^{2 \times 2}$ is an identity matrix.

In Eq. 13,,$\overline{\mathbf{u}}_{0}$ and $\overline{\mathbf{u}}$ include the elastic degrees of freedom fixed to $\mathcal{B}$ in order to correctly model the structure's mass properties. Since the elastic degrees of freedom fixed to $\mathcal{B}$ are typically set equal to zero, $\overline{\mathbf{u}}$ can be expressed in terms of the vector of independent nodal displacements $\mathbf{u} \in \mathbb{R}^{n}$ from Eq. (1) using the Boolean matrix $\mathbf{B} \in \mathbb{R}^{\left(n+n_{f}\right) \times n}$ that applies the boundary conditions that fix the structure to $\mathcal{B}$; i.e., $\overline{\mathbf{u}}=\mathbf{B u}$ [35]. Additional constraints are then required to specify any boundary conditions on the system in $\mathcal{I}$; see Sec. III.D.

Using Eq. (4), $\mathbf{r}_{i}$ can then be expressed in terms of the reduced basis $\boldsymbol{\Phi}$ as

$$
\mathbf{r}_{i}=\mathbf{R}+\mathbf{T L}_{i}\left(\overline{\mathbf{u}}_{0}+\mathbf{B \Phi} \hat{\mathbf{u}}\right)
$$

where $\hat{\mathbf{u}}$ is the vector of reduced generalized coordinates. The FOM is obtained when $\boldsymbol{\Phi}$ is an identity matrix.

Finally, differentiating Eq. (15) with respect to time in $\mathcal{I}$ results in the following expression for the velocity of node $i$ :

$$
\dot{\mathbf{r}}_{i}=\underbrace{\left[\begin{array}{lll}
\mathbf{I}_{2} & \mathbf{T}_{\psi} \mathbf{L}_{i}\left(\overline{\mathbf{u}}_{0}+\mathbf{B \Phi} \hat{\mathbf{u}}\right) & \mathbf{T} \mathbf{L}_{i} \mathbf{B \Phi}
\end{array}\right]}_{\mathbf{E}_{i}} \underbrace{\left[\begin{array}{c}
\dot{\mathbf{R}} \\
\dot{\psi} \\
\dot{\hat{\mathbf{u}}}
\end{array}\right]}_{\dot{\mathbf{q}}}
$$

where $\mathbf{E}_{i} \in \mathbb{R}^{2 \times n_{q}}$ is a configuration-dependent matrix, $\mathbf{q} \in \mathbb{R}^{n_{q}}$ is the generalized coordinate vector for the FFR used in the formulation of the Lagrangian equations of motion in Sec. III.D, and $n_{q}=3+r$.

\section{B. Kinetic Energy and Inertia Forces}

The inertia forces are derived from the kinetic energy using Lagrange's equations. Under the lumped mass approximation, the kinetic energy is

$$
\mathcal{T}=\frac{1}{2} \sum_{i=1}^{n_{n}} m_{i} \dot{\mathbf{r}}_{i}^{T} \dot{\mathbf{r}}_{i}
$$

where $n_{n}$ is the number of nodes in the finite element model and $m_{i}$ is the mass lumped at node $i$. Several methods exist for computing lumped masses from a consistent mass matrix; for an overview, see [37]. In this paper, the lumped masses are computed by neglecting rotational inertia terms and using the row sum method from [37]. Using Eq. (16] then allows Eq. (17) to be written as

$$
\mathcal{T}=\frac{1}{2} \dot{\mathbf{q}}^{T} \underbrace{\left(\sum_{i=1}^{n_{n}} m_{i} \mathbf{E}_{i}^{T} \mathbf{E}_{i}\right)}_{\mathbf{M}^{F}} \dot{\mathbf{q}}
$$

where $\mathbf{M}^{F} \in \mathbb{R}^{n_{q} \times n_{q}}$ is the configuration-dependent symmetric positive definite FFR mass matrix given by

$$
\mathbf{M}^{F}=\left[\begin{array}{lll}
\mathbf{M}_{R R} & \mathbf{M}_{R \psi} & \mathbf{M}_{R u} \\
& \mathbf{M}_{\psi \psi} & \mathbf{M}_{\psi u} \\
\operatorname{sym} & & \mathbf{M}_{u u}
\end{array}\right]
$$


The components of $\mathbf{M}^{F}$ are

$$
\begin{aligned}
& \mathbf{M}_{R R}=m \mathbf{I}_{2} \\
& \mathbf{M}_{R \psi}=\mathbf{T}_{\psi}\left(\overline{\mathbf{S}} \overline{\mathbf{u}}_{0}+\overline{\mathbf{S}} \mathbf{B} \boldsymbol{\Phi} \hat{\mathbf{u}}\right) \\
& \mathbf{M}_{R u}=\mathbf{T} \overline{\mathbf{S}} \mathbf{B} \boldsymbol{\Phi} \\
& \mathbf{M}_{\psi \psi}=\overline{\mathbf{u}}_{0}^{T} \mathbf{S}_{u u} \overline{\mathbf{u}}_{0}+2 \overline{\mathbf{u}}_{0}^{T} \mathbf{S}_{u u} \mathbf{B} \boldsymbol{\Phi} \hat{\mathbf{u}}+\hat{\mathbf{u}}^{T} \boldsymbol{\Phi}^{T} \mathbf{B}^{T} \mathbf{S}_{u u} \mathbf{B} \boldsymbol{\Phi} \hat{\mathbf{u}} \\
& \mathbf{M}_{\psi u}=\overline{\mathbf{u}}_{0}^{T} \widetilde{\mathbf{S}} \mathbf{B} \boldsymbol{\Phi}+\hat{\mathbf{u}}^{T} \boldsymbol{\Phi}^{T} \mathbf{B}^{T} \widetilde{\mathbf{S}} \mathbf{B} \boldsymbol{} \\
& \mathbf{M}_{u u}=\boldsymbol{\Phi}^{T} \mathbf{B}^{T} \mathbf{S}_{u u} \mathbf{B} \boldsymbol{\Phi}
\end{aligned}
$$

where orthogonality is used to simplify Eqs. 20d and 20f and the following are constant inertia terms:

$$
m=\sum_{i=1}^{n_{n}} m_{i}, \quad \overline{\mathbf{S}}=\sum_{i=1}^{n_{n}} m_{i} \mathbf{L}_{i}, \quad \widetilde{\mathbf{S}}=\sum_{i=1}^{n_{n}} m_{i} \mathbf{L}_{i}^{T}\left[\begin{array}{cc}
0 & 1 \\
-1 & 0
\end{array}\right] \mathbf{L}_{i}, \quad \mathbf{S}_{u u}=\sum_{i=1}^{n_{n}} m_{i} \mathbf{L}_{i}^{T} \mathbf{L}_{i}
$$

In the Lagrangian approach, the inertia forces are computed by differentiating the kinetic energy, as follows:

$$
\frac{\mathrm{d}}{\mathrm{d} t}\left(\frac{\partial \mathcal{T}}{\partial \dot{\mathbf{q}}}\right)^{T}-\left(\frac{\partial \mathcal{T}}{\partial \mathbf{q}}\right)^{T}=\mathbf{M}^{F} \ddot{\mathbf{q}}-\mathbf{G}^{F}
$$

where $\mathbf{G}^{F}$ is the vector of gyroscopic and Coriolis forces (often referred to as the quadratic velocity vector [35]) given by

$$
\mathbf{G}^{F}=-\dot{\mathbf{M}}^{F} \dot{\mathbf{q}}+\frac{1}{2}\left[\frac{\partial}{\partial \mathbf{q}}\left(\dot{\mathbf{q}}^{T} \mathbf{M}^{F} \dot{\mathbf{q}}\right)\right]^{T}=\left[\begin{array}{c}
\mathbf{G}_{X}^{F} \\
\mathbf{G}_{\psi}^{F} \\
\mathbf{G}_{u}^{F}
\end{array}\right]
$$

with components

$$
\begin{aligned}
& \mathbf{G}_{X}^{F}=\mathbf{T}\left(\overline{\mathbf{S}} \overline{\mathbf{u}}_{0}+\overline{\mathbf{S}} \mathbf{B} \boldsymbol{\Phi} \hat{\mathbf{u}}\right) \dot{\psi}^{2}-2 \mathbf{T}_{\psi} \overline{\mathbf{S}} \mathbf{B} \boldsymbol{\Phi} \dot{\hat{\mathbf{u}}} \dot{\psi} \\
& \mathbf{G}_{\psi}^{F}=-2\left(\overline{\mathbf{u}}_{0}^{T} \mathbf{S}_{u u} \mathbf{B} \boldsymbol{\Phi}+\hat{\mathbf{u}}^{T} \boldsymbol{\Phi}^{T} \mathbf{B}^{T} \mathbf{S}_{u u} \mathbf{B} \boldsymbol{\Phi}\right) \dot{\hat{\mathbf{u}}} \dot{\psi} \\
& \mathbf{G}_{u}^{F}=\left(\boldsymbol{\Phi}^{T} \mathbf{B}^{T} \mathbf{S}_{u u} \overline{\mathbf{u}}_{0}+\boldsymbol{\Phi}^{T} \mathbf{B}^{T} \mathbf{S}_{u u} \mathbf{B} \boldsymbol{\Phi} \hat{\mathbf{u}}\right) \dot{\psi}^{2}+2 \boldsymbol{\Phi}^{T} \mathbf{B}^{T} \widetilde{\mathbf{S}} \mathbf{B} \boldsymbol{\Phi} \dot{\hat{\mathbf{u}}} \dot{\psi}
\end{aligned}
$$

\section{Strain Energy and Elastic Forces}

In the FFR formulation, the strain energy is only a function of the elastic degrees of freedom; i.e.,

$$
\mathcal{U}=\mathcal{U}(\mathbf{B u}) \approx \mathcal{U}(\mathbf{B} \Phi \hat{\mathbf{u}})
$$

For geometrically nonlinear deformations, the strain energy defines a potential. Hence, the elastic generalized forces are obtained from the gradient of the strain energy and are given by

$$
\mathbf{F}_{n l}^{F}(\mathbf{q})=\left(\frac{\partial \mathcal{U}}{\partial \mathbf{q}}\right)^{T}=\left[\begin{array}{c}
\mathbf{0}_{2 \times 1} \\
0 \\
\boldsymbol{\Phi}^{T} \mathbf{B}^{T} \mathbf{F}_{n l}(\mathbf{B \Phi} \hat{\mathbf{u}})
\end{array}\right]
$$

where a boldface zero denotes a null matrix or vector with dimensions defined by the corresponding subscript; e.g., $\mathbf{0}_{2 \times 1}$ denotes the $2 \times 1$ null vector. Evaluating the Jacobian of Eq. 25) then results in the following expression for the FFR tangent stiffness matrix:

$$
\mathbf{K}_{T}^{F}(\mathbf{q})=\frac{\partial \mathbf{F}_{n l}^{F}}{\partial \mathbf{q}}=\left[\begin{array}{ccc}
\mathbf{0}_{2 \times 2} & \mathbf{0}_{2 \times 1} & \mathbf{0}_{2 \times r} \\
& 0 & \mathbf{0}_{1 \times r} \\
\operatorname{sym} & & \boldsymbol{\Phi}^{T} \mathbf{B}^{T} \mathbf{K}_{T}(\mathbf{B} \boldsymbol{\Phi} \hat{\mathbf{u}}) \mathbf{B} \boldsymbol{\Phi}
\end{array}\right]
$$

where $\mathbf{K}_{T}$ is given by Eq. (2). 
Equations (25) and (26) suffer from the computational bottleneck discussed in Sec. II.C. To alleviate this computational bottleneck, Eqs. (25) and (26) are hyper-reduced using ECSW; i.e., $\boldsymbol{\Phi}^{T} \mathbf{B}^{T} \mathbf{F}_{n l}(\mathbf{B} \boldsymbol{\Phi} \hat{\mathbf{u}})$ and $\boldsymbol{\Phi}^{T} \mathbf{B}^{T} \mathbf{K}_{T}(\mathbf{B} \boldsymbol{\Phi} \hat{\mathbf{u}}) \mathbf{B} \boldsymbol{\Phi}$ are approximated by Eq. (7) and the Jacobian of Eq. (7), respectively. The hyper-reduced FFR-ROM inherits all of the useful properties associated with ECSW, including its preservation of the Lagrangian structure of the full-order finite element model.

\section{Equations of Motion}

The equations of motion are derived from Lagrange's equations, as follows:

$$
\begin{aligned}
\frac{\mathrm{d}}{\mathrm{d} t}\left(\frac{\partial \mathcal{T}}{\partial \dot{\mathbf{q}}}\right)^{T}-\left(\frac{\partial \mathcal{T}}{\partial \mathbf{q}}\right)^{T}+\left(\frac{\partial \mathcal{U}}{\partial \mathbf{q}}\right)^{T}+\mathbf{C}_{q}^{T} \lambda=\mathbf{F}_{e x t}^{F} \\
\mathbf{C}(\mathbf{q})=\mathbf{0}_{n_{\lambda} \times 1}
\end{aligned}
$$

where $\mathbf{C}(\mathbf{q}) \in \mathbb{R}^{n_{\lambda}}$ is the holonomic constraint vector, $\mathbf{C}_{q}=\partial \mathbf{C} / \partial \mathbf{q} \in \mathbb{R}^{n_{\lambda} \times n_{q}}$ is the constraint gradient matrix, $\lambda \in \mathbb{R}^{n_{\lambda}}$ is the vector of Lagrange multipliers, $n_{\lambda}$ is the number of constraint equations, and $\mathbf{F}_{\text {ext }}^{F} \in \mathbb{R}^{n_{q}}$ is the vector of external generalized forces. The holonomic constraint vector enforces any boundary conditions on the system in $\mathcal{I}$. Note that these constraints are expressed in the reduced basis $\boldsymbol{\Phi}$, which may necessitate a transformation of the physical constraints. Using Eqs. 21] and (25) then allows Eq. 27] to be rewritten as

$$
\begin{aligned}
\mathbf{M}^{F} \ddot{\mathbf{q}}-\mathbf{G}^{F}+\mathbf{F}_{n l}^{F}(\mathbf{q})+\mathbf{C}_{q}^{T} \lambda & =\mathbf{F}_{e x t}^{F} \\
\mathbf{C}(\mathbf{q}) & =\mathbf{0}_{n_{\lambda} \times 1}
\end{aligned}
$$

which are the equations of motion for the FFR-ROM.

\section{Elastica Catapult}

This section uses POD-ECSW and a FFR to develop a ROM for the elastica catapult [7] capable of describing static and dynamic behavior across a wide-range of loading parameters. It is organized as follows: Sec. IV.A sets up the problem and uses quasi-static simulations to illustrate the snap-back phenomenon. Basis selection and hyper-reduction are then addressed in Sec. IV.B. Finally, reduced-order modeling for the dynamic snap-back problem is studied in Sec. IV.C

\section{A. Problem Setup and Snap-Back}

The elastica catapult consists of a slender clamped-free plane rod rotating in a uniform gravitational field; see Fig. 1. The rod is prismatic, homogeneous, and linear-elastic with bending stiffness $E I$, length $\ell$, mass density $\rho$, and a rectangular cross-section of breadth $b$ and height $h$. The parameter $E$ is the elastic modulus and $I=b h^{3} / 12$ is the second moment of area. The material and geometric properties are taken from [7] and listed in Table 1. A point mass $\mu$ is attached at the free end, resulting in a tip load $P=\mu g$ where $g$ is the gravitational acceleration. The unit basis vectors $\{\hat{\mathbf{X}}, \hat{\mathbf{Y}}\}$ define the inertial reference frame $\mathcal{I}$. Gravity acts anti-parallel to $\hat{\mathbf{Y}}$, and the clamp is inclined at an angle $\alpha$ with respect to the gravity direction. The clamp rotates with respect to $\mathcal{I}$ and defines a rotating body reference frame $\mathcal{B}$ with unit basis vectors $\{\hat{\mathbf{x}}, \hat{\mathbf{y}}\} . \hat{\mathbf{x}}$ is parallel to, and the arc length parameter $X$ measures position along, the rod's undeformed neutral axis (the blue dashed line). Elastic deformations are measured with respect to $\mathcal{B}$ using a Total Lagrangian description, and $\theta(X)$ denotes the cross-sectional rotation with respect to $\mathcal{B}$. The deformation of the rod is due to the rotation of the clamp and the action of gravity.

The rod is discretized using 32 geometrically exact $C^{0}$ beam elements in the Total Lagrangian description. The beam elements are derived from the geometrically exact strain-displacement relation for a plane Timoshenko beam [38] using linear shape functions and the slender beam limit of MacNeal's residual bending flexibility (RBF) correction [39]. The formulation models large deformation lead-lag motion of a rotating beam, and is capable of modeling nonlinear phenomena, including snap-back, so long as the strains remain small. A similar formulation for flapping motion is presented in Appendix 2 of [40], but it does not correct for shear locking during linear curvature deformations [39]. The number of elements is based on the results of a mesh convergence study. The resulting finite element model, the FOM, has 96 independent elastic degrees of freedom and constitutes a reference solution. Each node in the FOM has three degrees of freedom: an axial displacement, a bending displacement, and a cross-sectional rotation. The FOM model is 


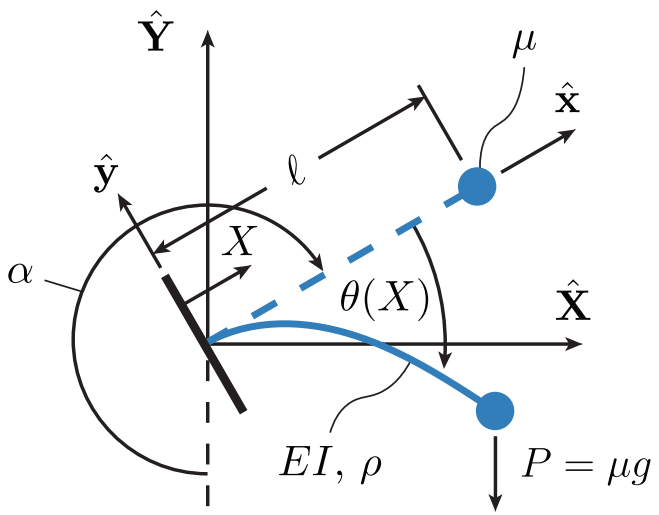

Fig. 1 Elastica catapult. Dashed and solid lines denote the undeformed and deformed configurations, respectively. The rotating clamp is inclined at an angle $\alpha$ with respect to the gravity direction. The deformation is due to the rotation of the clamp and gravity.

Table 1 Material and geometric properties for the elastica catapult from [7]

\begin{tabular}{ll}
\hline \hline Elastic modulus $E$ & $2350 \mathrm{MPa}$ \\
Mass density $\rho$ & $1180 \mathrm{~kg} / \mathrm{m}^{3}$ \\
Cross-sectional breadth $b$ & $25 \mathrm{~mm}$ \\
Cross-sectional height $h$ & $3 \mathrm{~mm}$ \\
Length $\ell$ & $345 \mathrm{~mm}$ \\
Mass-proportional damping coefficient $a$ & $10^{-2} \mathrm{~s}^{-1}$ \\
Stiffness-proportional damping coefficient $b$ & $5 \times 10^{-3} \mathrm{~s}$ \\
\hline \hline
\end{tabular}

extensible, whereas [7] assumes inextensibility for quasi-static and some dynamic solutions. Extensibility effects are negligible for the quasi-static problem, but in general must be accounted for in the dynamic problem [7].

Quasi-static analyses are conducted using a version of Crisfield's arc length solver [41] modified for the elastica catapult. Specifically, load increments are replaced by increments of the clamp inclination angle, and the residual and the Jacobian are modified accordingly. Quasi-static analyses neglect the mass of the beam; i.e., the only external force is due to the gravitational force acting on the tip mass. Unless otherwise specified, the arc length solver uses a fixed arc length of $10^{-3}$ and the Newton-Rhapson iterations are considered converged at an absolute tolerance of $10^{-4}$.

When the tip load $P$ exceeds the Euler buckling load $P_{c r}=\pi^{2} E I /\left(4 \ell^{2}\right)$, multiple equilibria exist for some clamp inclination angles. Upon reaching an unstable equilibrium point, the beam dynamically snaps to a new stable equilibrium configuration. This phenomenon is illustrated in Fig. 2 which depicts the tip rotations as a function of the clamp inclination angle for various values of $P / P_{c r}$. The results show excellent agreement with Fig. 2 in [7].

Based on Fig. 2, the clamp inclination angle $\alpha_{S}$ at the onset of the snap denotes the transition from quasi-static behavior to dynamic behavior. As a result, to accurately model the transient dynamics during snap-back, a ROM must accurately approximate the clamp inclination angle $\alpha_{S}$ at the onset of the snap. Figure 3 plots $\alpha_{S}$ as a function of load ratio $P / P_{c r} \in[1,5]$ and shows excellent agreement with Fig. 3 from [7]. The trend is that $\alpha_{S}$ increases as $P / P_{c r}$ increases; larger loads result in larger deformations which postpone the onset of the snap. The results in Fig. 3 are useful for predicting the onset of the snap in dynamic simulations.

\section{B. Evaluation of Reduced-Order Model}

The convergence of the POD-Galerkin approach is first studied to establish a baseline ROM. To evaluate the POD basis $\Phi$, the snapshot matrix is assembled from the displacements corresponding to 1000 equally spaced load increments from a nonlinear static analysis of the FOM with a transverse tip load of magnitude $P / P_{c r}=5$. As is standard, the reduced basis $\Phi$ then consists of the first $r$ left singular vectors of the snapshot matrix; see Sec. II.B. 


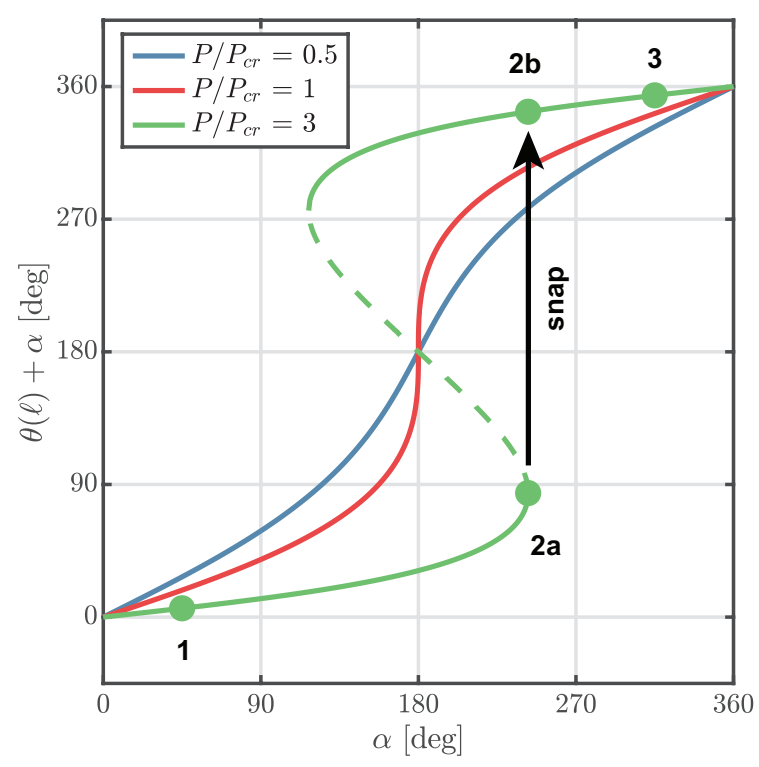

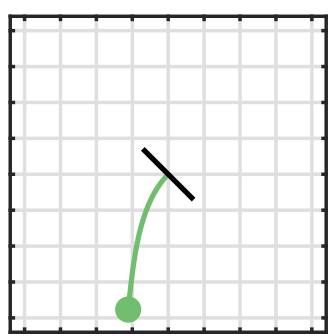

(b) $\alpha_{1}=45^{\circ}$

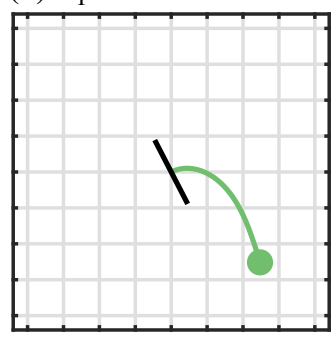

(d) $\alpha_{2 b} \approx 242.6^{\circ}$

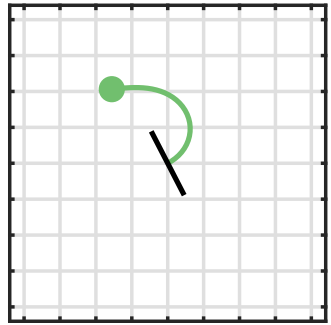

(c) $\alpha_{2 a} \approx 242.6^{\circ}$

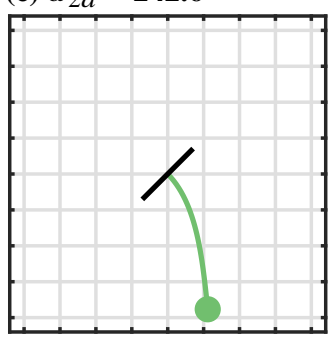

(e) $\alpha_{3}=315^{\circ}$

(a) Tip rotations

Fig. 2 Quasi-static analysis of the elastica catapult demonstrating snap-back for $\mathbf{P} / \mathbf{P}_{\mathrm{cr}}>1$. (a) plots tip rotations as a function of clamp inclination angle for various values of $P / P_{c r}$. Solid and dashed lines denote stable and unstable equilibrium configurations, respectively. (b)-(e) plot deflected shapes corresponding to equilibrium configurations pre- and post-snap.

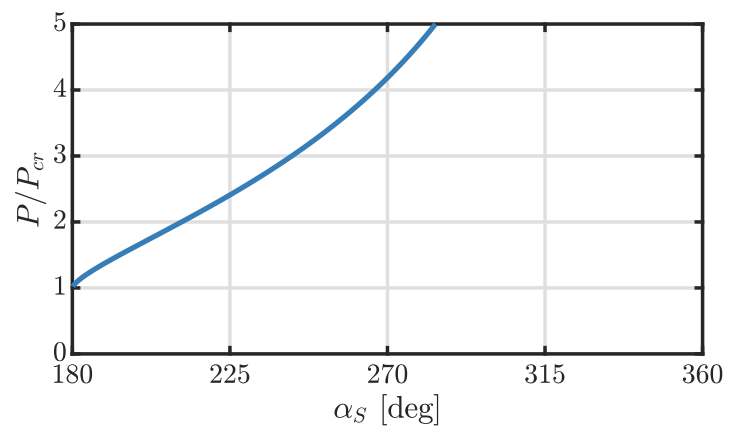

Fig. 3 Clamp inclination angle at snap-back as a function of $\mathbf{P} / \mathbf{P}_{\mathrm{cr}}$.

The results of a modal convergence study then show that 16 POD modes accurately approximate the quasi-static response of the elastica catapult for a wide range of load ratios. To illustrate this, consider Fig. 4 which plots tip rotations as a function of clamp inclination angle for various values of $r$ and $P / P_{c r}$. Fewer than 8 modes are required for convergence when $P / P_{c r}=0.5$, and 12 modes are required when $P / P_{c r}=1$. In contrast, 14-16 modes are required when $P / P_{c r}=3$, and $r$ must be further increased as $P / P_{c r}$ is further increased. Since a 16 mode POD-Galerkin ROM accurately approximates the FOM for $P / P_{c r} \in[0,3], 16$ modes are used in what follows. Thus, the Galerkin projection reduces the dimensionality of the elastic coordinates by nearly $85 \%$ compared to the FOM.

The POD-Galerkin ROM is then further reduced using the ECSW hyper-reduction method. Figure 5 plots the number of elements in the reduced mesh as a function of the error tolerance $\epsilon \in[0,1]$. The results show that a reduced mesh with as few as 9 elements (an approximately $72 \%$ reduction in the mesh size) provides no appreciable loss in accuracy compared to the FOM. Specifically, the minimum error tolerance for a 9 element reduced mesh is $\epsilon=0.0053$; i.e., a 9 element mesh results in an approximately $0.53 \%$ error over the training data set. Figure 6 then plots the reduced mesh and corresponding element weights $\xi_{e}^{*}$ for $\epsilon=0.0053$. In the reduced mesh, a higher density of elements are 

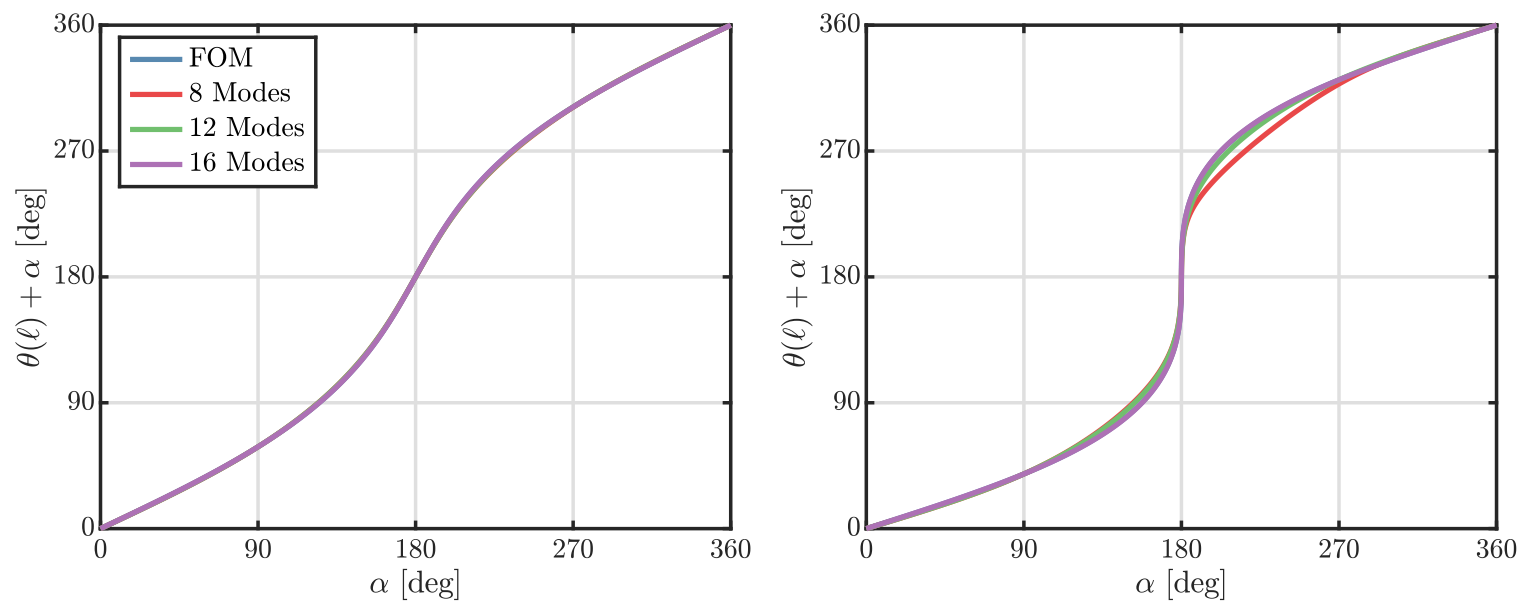

(a) $\mathbf{P} / \mathbf{P}_{\text {cr }}=0.5$

(b) $\mathbf{P} / \mathbf{P}_{\text {cr }}=\mathbf{1}$

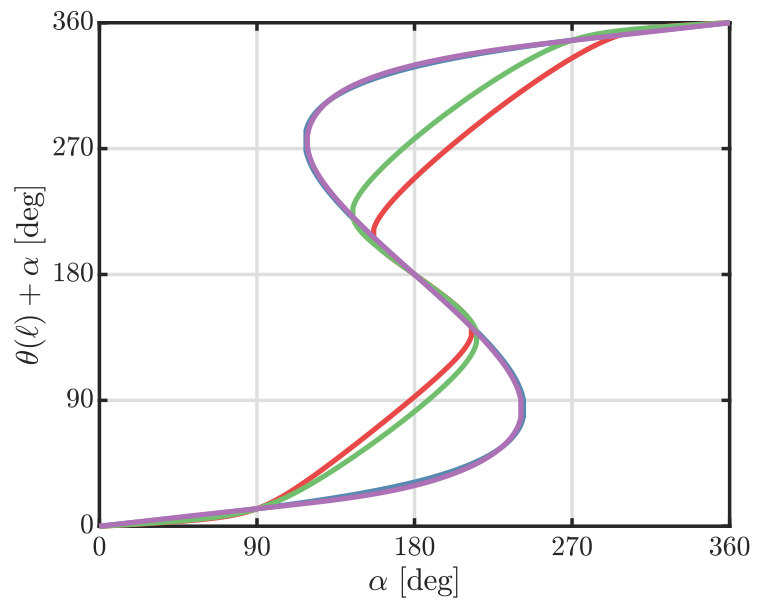

(c) $\mathbf{P} / \mathbf{P}_{\text {cr }}=\mathbf{3}$

Fig. 4 Modal convergence of POD-Galerkin ROMs for various values of $\mathbf{P} / \mathbf{P}_{\mathrm{cr}}$.

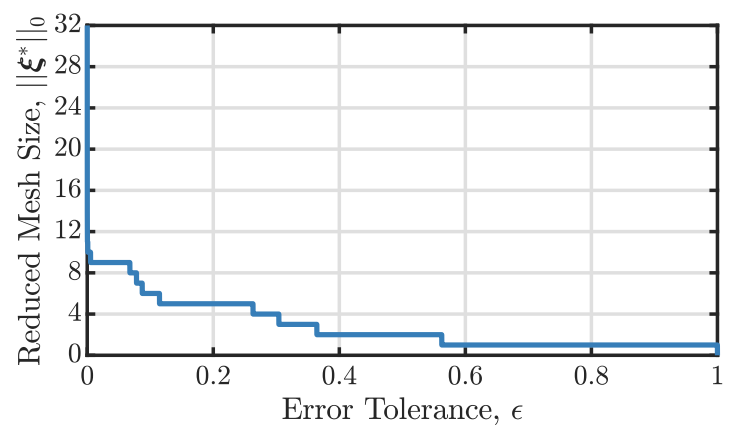

Fig. 5 Number of elements required to achieve a specified error tolerance with ECSW.

required to accurately reconstruct the response near the clamp, and approximately $87 \%$ of the element-level contributions are associated with the elements with the 6 largest weights. As shown next, the reduced mesh with 9 elements does not appreciably change the accuracy of the ROM. Hence, this reduced mesh is used in subsequent simulations.

To demonstrate the accuracy of the POD-ECSW ROM, the tip rotations as a function of clamp inclination angle for the POD-ECSW ROM and the FOM are compared in Fig. 7 for $P / P_{c r}=0.5, P / P_{c r}=1$, and $P / P_{c r}=3$. Based on 


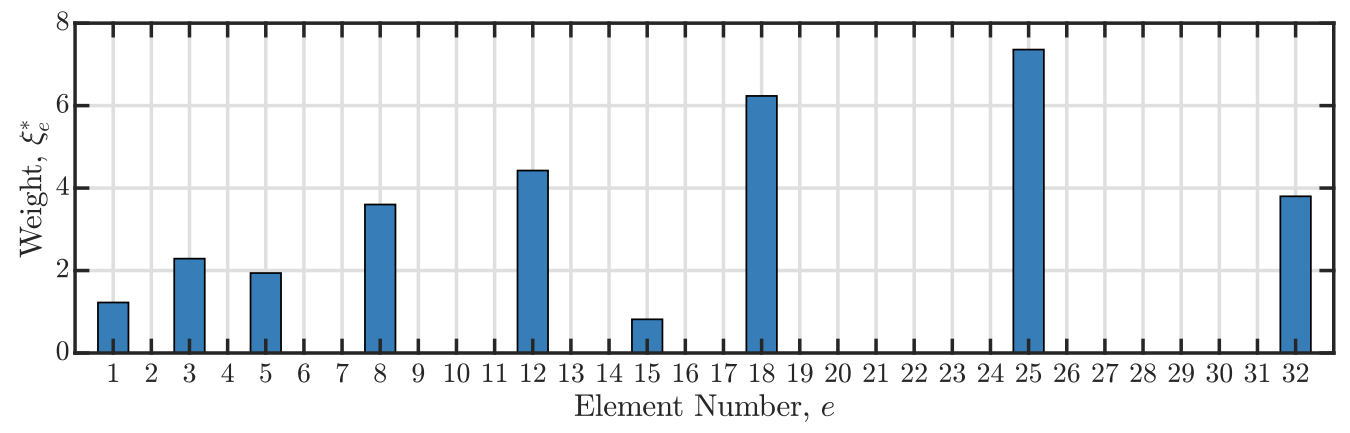

Fig. 6 POD-ECSW reduced mesh with 9 elements corresponding to $\epsilon=0.0053$.
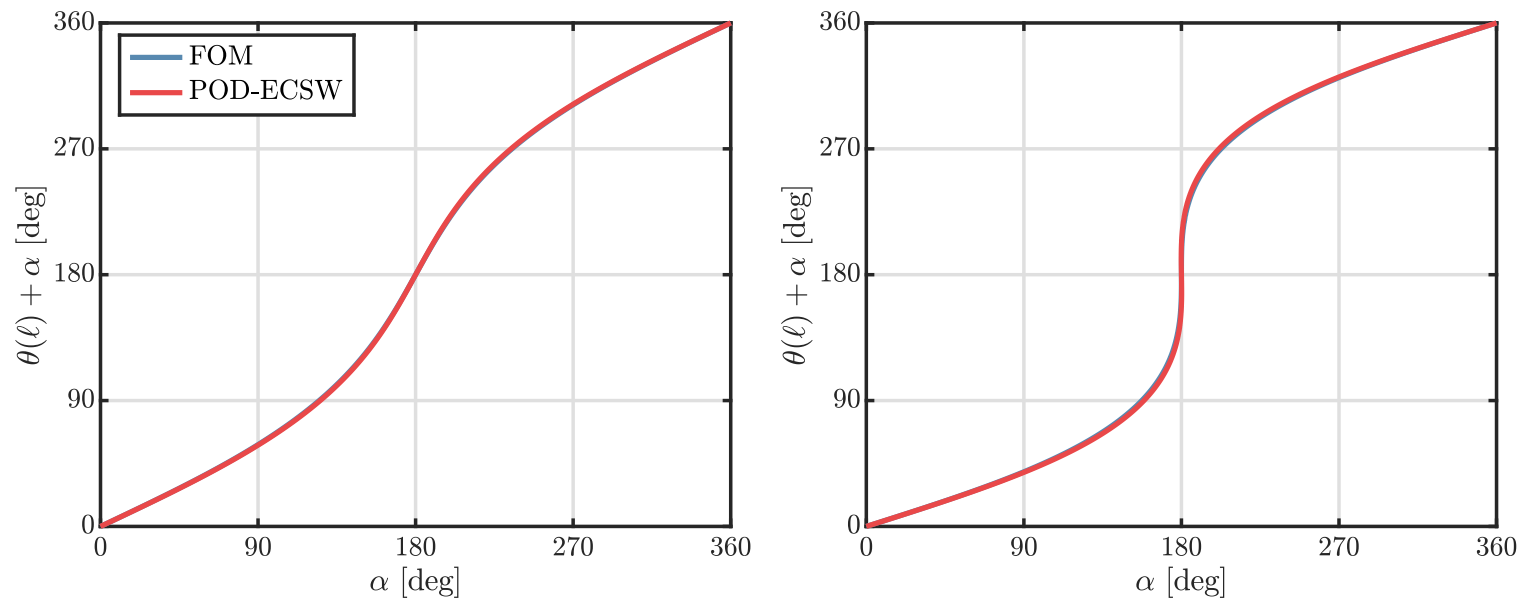

(a) $\mathbf{P} / \mathbf{P}_{\text {cr }}=0.5$

(b) $\mathbf{P} / \mathbf{P}_{\text {cr }}=\mathbf{1}$

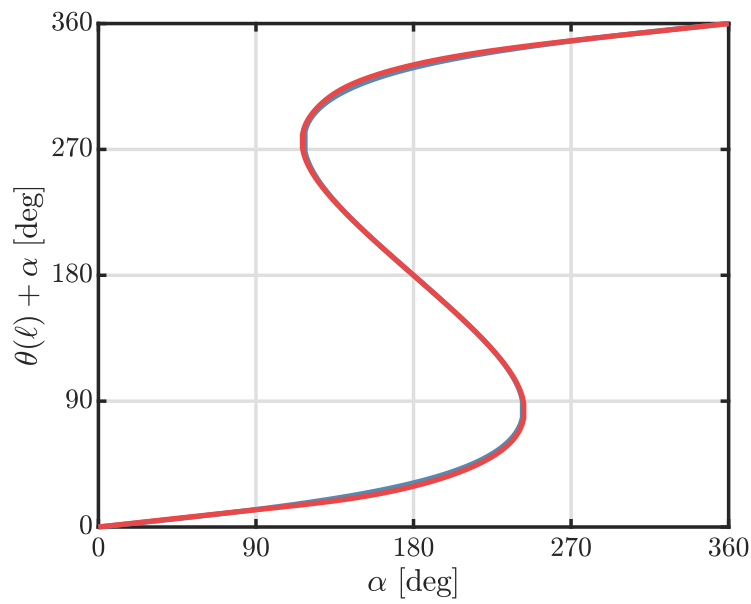

(c) $\mathbf{P} / \mathbf{P}_{\text {cr }}=\mathbf{3}$

Fig. 7 Comparison between POD-ECSW and the FOM for various values of $P / P_{c r}$.

Fig. 7 the POD-ECSW solution is almost indistinguishable from the FOM solution for a wide range of values of $P / P_{c r}$. The reduced mesh correctly models the snap-back effect because each element in the reduced mesh is individually capable of modeling snap-back. Altogether, POD-ECSW significantly reduces dimensionality and complexity without any appreciable loss in accuracy. 


\section{Dynamic Snap-Back}

To conclude the study of the elastica catapult, results are presented for 2 load cases: $P / P_{c r}=1.142$ and $P / P_{c r}=1.815$. For each case, results for the 16 mode POD-Galerkin and 16 mode, 9 element POD-ECSW ROMs from Sec.IV.B are compared with results for the FOM. A FFR from Sec. III is used to simulate the clamp rotation. The approach used for the dynamic simulations is described in Sec.IV.C.1 and results are presented in Sec.IV.C.2 All of the simulations in this section use a time step $\Delta t=10^{-4} \mathrm{~s}$ and a final time $t_{f}=45 \mathrm{~s}$.

\section{Approach to Dynamic Simulations}

Following [7], the dynamic simulations time-integrate the equations of motion for the FOM and ROMs using the two-step solution process outlined below. In both steps, the absolute convergence tolerance for the Newton-Rhapson iterations is set to $10^{-8}$.

1) A nonlinear static solver is used to find the initial deformed configuration due to the weight of the tip mass and the beam's self-weight when $\alpha=0$. For each ROM, this step is carried out on the ROM directly, as opposed to mapping the initial deformed configuration from the FOM to the reduced basis. This guarantees that the initial conditions are static equilibrium configurations for the ROMs.

2) The equations of motion are time-integrated using a MATLAB ${ }^{\circledR}$ implementation of the implicit generalized- $\alpha$ method of Arnold and Brüls [42]. Dynamic simulations assume that at time $t<0$, the beam is in static equilibrium with the initial conditions from the previous step. At time $t=0$, a constant angular velocity $\dot{\alpha}=2 \pi / t_{f}$ is applied to the clamp where $t_{f}$ is the simulation duration. The clamp rotates at $\dot{\alpha}$ for the duration of the simulation.

For the FFR formulation, the origin and orientation of the body reference frame are specified as $\mathbf{R}=\mathbf{0}_{2 \times 1}$ (i.e., the origin is at the clamp) and $\psi=3 \pi / 2-\dot{\alpha} t$, respectively. These constraints on $\mathbf{R}$ and $\psi$ are enforced via Lagrange multipliers in the equations of motion [Eq. [28]].

Dynamic analyses include the beam's self-weight when evaluating the gravitational forces on the system. The FOM evaluates the inertial and gravitational forces using a consistent mass formulation. The ROMs evaluate the inertial and gravitational forces using the lumped mass formulation from Sec. III The gravitational forces and corresponding load stiffness matrix in the lumped mass formulation are

$$
\begin{aligned}
& \mathbf{F}_{e x t}^{F}=-\left(\frac{\partial \mathcal{U}_{g}}{\partial \mathbf{q}}\right)^{T}=-g\left[\begin{array}{c}
m \mathbf{e}_{2} \\
\mathbf{e}_{2}^{T} \mathbf{T}_{\psi} \overline{\mathbf{S}}\left(\overline{\mathbf{u}}_{0}+\mathbf{B} \boldsymbol{\Phi} \hat{\mathbf{u}}\right) \\
\boldsymbol{\Phi}^{T} \mathbf{B}^{T} \overline{\mathbf{S}}^{T} \mathbf{T}^{T} \mathbf{e}_{2}
\end{array}\right] \\
& \mathbf{K}_{L}^{F}=\frac{\partial \mathbf{F}_{e x t}^{F}}{\partial \mathbf{q}}=-g\left[\begin{array}{ccc}
\mathbf{0}_{2 \times 2} & \mathbf{0}_{2 \times 1} & \mathbf{0}_{2 \times r} \\
\mathbf{0}_{1 \times 2} & -\mathbf{e}_{2}^{T} \mathbf{T} \overline{\mathbf{S}}\left(\overline{\mathbf{u}}_{0}+\mathbf{B} \boldsymbol{\Phi} \hat{\mathbf{u}}\right) & \mathbf{e}_{2}^{T} \mathbf{T}_{\psi} \overline{\mathbf{S}} \mathbf{B \Phi} \\
\mathbf{0}_{r \times 2} & \boldsymbol{\Phi}^{T} \mathbf{B}^{T} \overline{\mathbf{S}}^{T} \mathbf{T}_{\psi}^{T} \mathbf{e}_{2} & \mathbf{0}_{r \times r}
\end{array}\right]
\end{aligned}
$$

where $\mathcal{U}_{g}$ is the gravitational potential energy. Equations (29) and (30) reflect that the magnitude of the gravitational force is constant, but its direction in the body reference frame is a function of the clamp inclination angle.

The implicit generalized- $\alpha$ method [43] is used for time-integration because of its user-controlled numerical dissipation. Numerical dissipation eliminates the high frequency numerical oscillations that arise in the solution of stiff differential equations and has a stabilizing effect on the dynamic tangent matrix in the presence of instabilities. Moreover, the infinite natural frequencies associated with the constraints in Eq. (28) introduce a weak numerical instability which can be eliminated through numerical dissipation [44]. In the generalized- $\alpha$ scheme, numerical dissipation is specified through the spectral radius at infinity $\rho_{\infty} \in[0,1]$. The authors' numerical experiments show that the accuracy of the simulations are not very sensitive to the amount of numerical dissipation, especially in the presence of viscous damping. As a result, $\rho_{\infty}=0.7$ is used in this paper. To avoid numerical ill-conditioning of the dynamic tangent matrix, the scaling approach of [45] is used, as recommended in [42].

Finally, as is done in [7], Rayleigh damping is incorporated into the dynamic simulations. The constant Rayleigh damping matrix for the FOM is

$$
\mathbf{D}_{R}=\alpha_{R} \mathbf{M}+\beta_{R} \mathbf{K}_{T}^{b}
$$

where $\alpha_{R}$ and $\beta_{R}$ are the mass- and stiffness-proportional damping coefficients from Table 1 and $\mathbf{K}_{T}^{b}=\partial \mathbf{F}_{n l}^{b} / \partial \mathbf{u}$ is the tangent bending stiffness matrix; i.e., $\mathbf{F}_{n l}^{b}$ is the bending contribution to the vector of internal elastic forces. For simplicity, $\mathbf{D}_{R}$ is evaluated once in the undeformed configuration. It follows that the constant Rayleigh damping matrix 

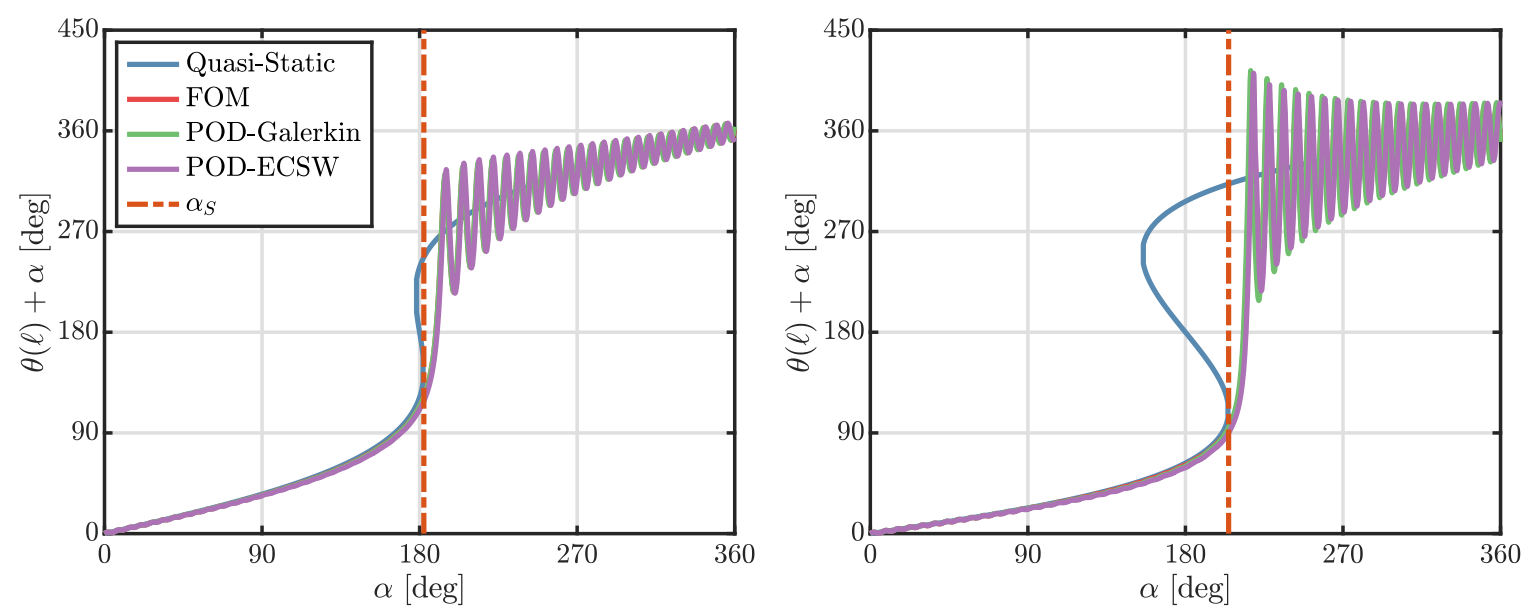

(a) $\mathbf{P} / \mathbf{P}_{\text {cr }}=1.142$

(b) $\mathbf{P} / \mathbf{P}_{\text {cr }}=1.815$

Fig. 8 Comparison between tip rotations for the POD-Galerkin ROM, POD-ECSW ROM, and the FOM for various values of $\mathbf{P} / \mathbf{P}_{\mathrm{cr}}$.

for the ROM is

$$
\mathbf{D}_{R}^{F}=\left[\begin{array}{ccc}
\mathbf{0}_{2 \times 2} & \mathbf{0}_{2 \times 1} & \mathbf{0}_{2 \times r} \\
& 0 & \mathbf{0}_{1 \times r} \\
\text { sym } & & \boldsymbol{\Phi}^{T} \mathbf{B}^{T} \mathbf{D}_{R} \mathbf{B} \boldsymbol{\Phi}
\end{array}\right]
$$

Whereas the numerical dissipation acts on both rigid and elastic states, Rayleigh damping is only applied to the elastic states. Rayleigh damping improves the convergence of the simulations.

\section{Dynamic Simulation Results}

The transient dynamics for $P / P_{c r}=1.142$ and $P / P_{c r}=1.815$ are depicted in Figs. $8 \mathrm{a}$ and $8 \mathrm{~b}$, respectively, for the case when the clamp rotates $360^{\circ}$ in $t_{f}=45 \mathrm{~s}$. Specifically, Figs. $8 \mathrm{a}$ and $8 \mathrm{~b}$ compare the tip rotations in the inertial frame for the ROMs and the FOM. The tip rotations clearly indicate snap-back. Before the snap, the response is approximately quasi-static; after the snap, there is a large amplitude transient oscillation that dissipates due to the Rayleigh damping. Not surprisingly, the initial amplitude of the transient oscillations increases as $P / P_{c r}$ increases. The quasi-static response of the FOM neglects the beam's self-weight; as a result, the quasi-static model underestimates $\alpha_{S}$. Adding self-weight in the dynamic simulations effectively increases the mass at the tip, resulting in larger deformations that postpone the onset of the snap (see Fig. 3). Overall, Figs. $8 \mathrm{a}$ and $8 \mathrm{~b}$ demonstrate that both ROMs show good qualitative agreement with the FOM.

Figures 9 and 10 then plot the root bending moments and tip mass accelerations for $P / P_{c r}=1.142$ and $P / P_{c r}=1.815$. Two metrics used for assessing the accuracy of the ROMs during snap-back are derived from these figures: the estimated clamp inclination angle at the onset of the snap $\bar{\alpha}_{S}$ and the maximum acceleration of the tip mass in the inertial frame $\|\mathbf{a}(\ell)\|_{2}$. The notation $\bar{\alpha}_{S}$ is used to distinguish this quantity from the quantity $\alpha_{S}$ predicted by the quasi-static simulations (Fig. 3). Following [7], $\bar{\alpha}_{S}$ is estimated by using linear interpolation to estimate the clamp inclination angle corresponding to the initial change in the sign of the root bending moment in Figs. 9a and 10a. The tip mass accelerations and root bending moments from Figs. 9 and 10 show generally good agreement with the FOM results.

The snap-back error metrics for $P / P_{c r}=1.142$ and $P / P_{c r}=1.815$ are summarized in Table 2 For both cases, the results show that the ROMs accurately predict when snap-back occurs and the amplitude of the resulting transient oscillations. The latter is expected because ECSW approximates energy-like quantities, and the amount of stored energy determines the amplitude of the subsequent oscillations. Additionally, the results show that the error increases with increasing $P / P_{c r}$, as expected, and that the POD-Galerkin ROM is consistently more accurate than the POD-ECSW ROM because it involves fewer approximations of the original FOM. The errors between the ROMs can be decreased by increasing the density of the elements in the reduced mesh, at the expense of added computational cost. Altogether, these results show that the POD-ECSW ROM likely provides sufficient accuracy for engineering design and analysis. 


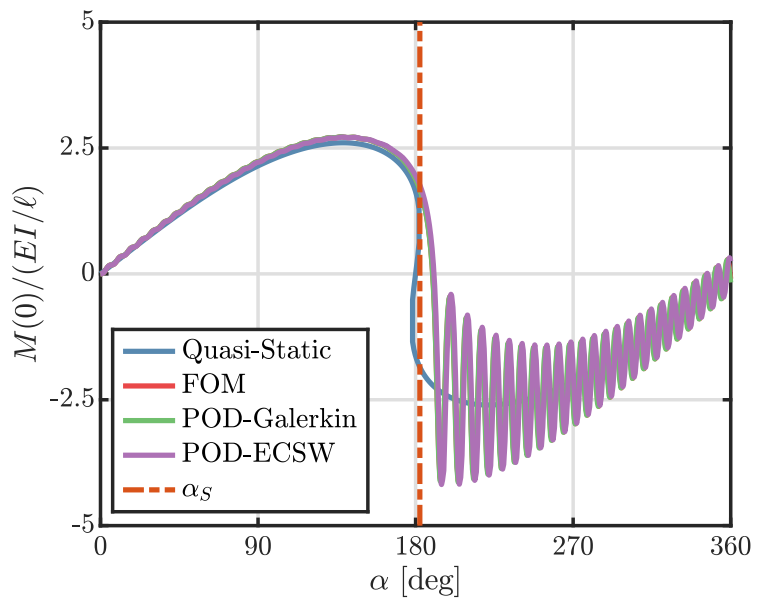

(a) Root bending moments

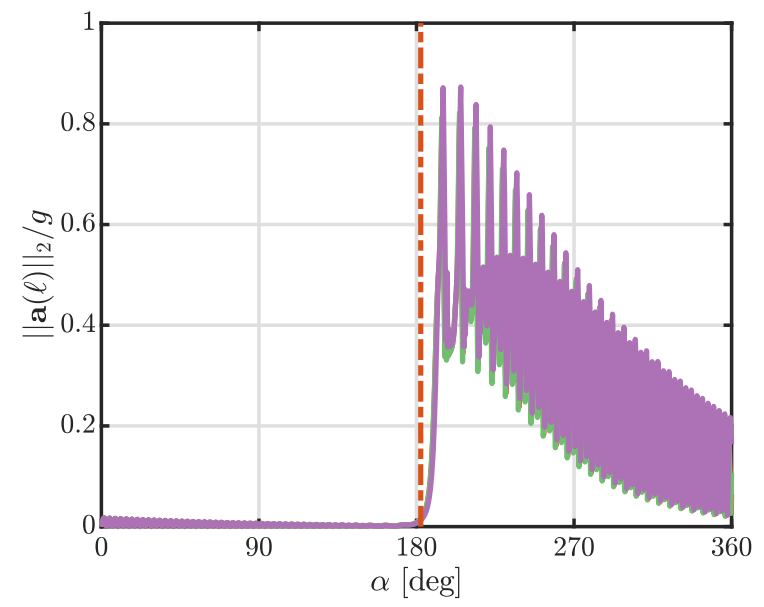

(b) Tip mass accelerations

Fig. 9 Comparison between (a) root bending moments and (b) tip mass accelerations for $\mathbf{P} / \mathbf{P}_{\mathrm{cr}}=1.142$.

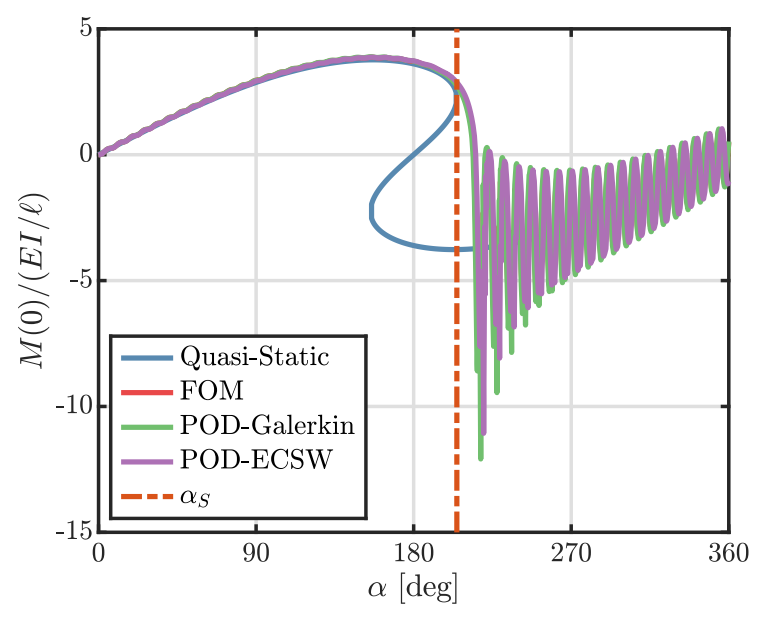

(a) Root bending moments

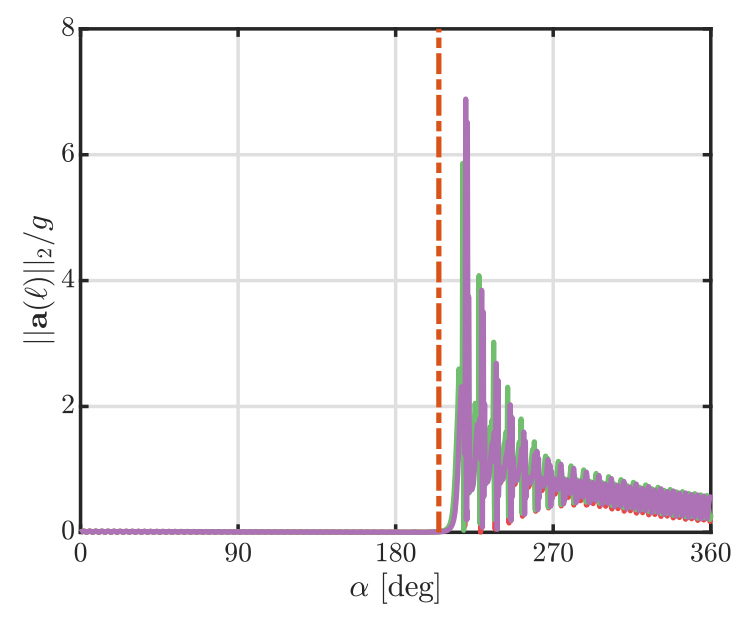

(b) Tip mass accelerations

Fig. 10 Comparison between (a) root bending moments and (b) tip mass accelerations for $\mathbf{P} / \mathbf{P}_{\mathrm{cr}}=1.815$.

Table 2 Error metrics for assessing ROM accuracy during snap-back. \% errors are relative to the FOM

\begin{tabular}{cccccc}
\hline \hline$P / P_{c r}$ & Model & $\bar{\alpha}_{S}[\mathrm{deg}]$ & \% error & $\max \left(\|\mathbf{a}(\ell)\|_{2}\right) / g$ & \% error \\
\hline \multirow{4}{*}{1.142} & POD-Galerkin & 189.71 & -0.028 & 0.822 & 1.24 \\
& POD-ECSW & 190.51 & 0.392 & 0.873 & 7.55 \\
& FOM & 189.76 & 0 & 0.812 & 0 \\
\hline \multirow{3}{*}{1.815} & POD-Galerkin & 213.93 & -0.188 & 5.863 & -5.76 \\
& POD-ECSW & 215.44 & 0.517 & 6.885 & 10.65 \\
& FOM & 214.33 & 0 & 6.222 & 0 \\
\hline \hline
\end{tabular}

\section{Conclusion}

Reduced-order modeling for a class of ultralight, packageable, and self-deployable spacecraft has been investigated. These spacecraft are characterized by geometrically nonlinear structural dynamics, large rigid body motions, and energy release during deployment. The proposed reduced-order modeling approach uses Galerkin projection and 
hyper-reduction applied to a geometrically nonlinear finite element model in a lumped mass, floating frame of reference (FFR). Proper orthogonal decomposition (POD) is used to find the reduced basis for the Galerkin projection, and energy-conserving sampling and weighting (ECSW) is used for hyper-reduction.

The proposed approach was demonstrated on a prototypical problem, the elastica catapult from Armanini et al. [7]. The elastica catapult's energy release during dynamic snap-back resembles the energy release during deployment and localized folding of ultralight spacecraft structures. As a result, it is a relevant benchmark problem for studying reduced-order modeling methods for ultralight, flexible spacecraft.

A full-order model (FOM) of the elastica catapult with 96 independent elastic degrees of freedom was developed using 32 geometrically exact $C^{0}$ beam elements capable of modeling large deformation lead-lag motion of a rotating beam. POD and ECSW were then used to reduce this FOM into a 16 mode, 9 element reduced-order model (ROM); i.e., a ROM with 16 reduced elastic degrees of freedom assembled from a reduced mesh with 9 elements. Three additional degrees of freedom -2 translations and 1 rotation - described the rigid body motion of the FFR. This resulted in a ROM approximately $20 \%$ of the size of the original FOM with significantly reduced computational complexity.

Quasi-static and dynamic simulations with a range of tip masses illustrated the capability of the ROM to model nonlinear phenomena like the large transient oscillations induced by dynamic snap-back. Two quantitative metrics the clamp inclination angle at snap-back and the maximum acceleration of the tip mass - were then used to assess the accuracy of the ROM during dynamic snap-back. Overall, good agreement was observed between the ROM and the FOM, and both metrics demonstrated that the ROM provided sufficient accuracy for engineering design and analysis.

Altogether, the proposed approach shows promise for reduced-order modeling of ultralight, flexible spacecraft. Future work entails applying the proposed approach to more complicated finite element models characteristic of an actual ultralight, flexible spacecraft, like the Caltech Space Solar Power Satellite [6].

\section{Acknowledgments}

M. A. Marshall was supported by a NASA Space Technology Research Fellowship. Financial support from the California Institute of Technology (Caltech) Space Solar Power Project is also gratefully acknowledged. The authors thank W. Keats Wilkie and F. Gianfranco Canales Escobedo for the many helpful discussions.

\section{References}

[1] Sawada, H., Mori, O., Okuizumi, N., Shirasawa, Y., Miyazaki, Y., Natori, M., Matunaga, S., Furuya, H., and Sakamoto, H., "Mission Report on The Solar Power Sail Deployment Demonstration of IKAROS," 52nd AIAA/ASME/ASCE/AHS/ASC Structures, Structural Dynamics and Materials Conference, Denver, Colorado, 2011. doi 10.2514/6.2011-1887

[2] Fernandez, J. M., Rose, G., Stohlman, O. R., Younger, C. J., Dean, G. D., Warren, J. E., Kang, J. H., Bryant, R. G., and Wilkie, K. W., "An Advanced Composites-Based Solar Sail System for Interplanetary Small Satellite Missions," 2018 AIAA Spacecraft Structures Conference, Kissimmee, Florida, 2018. doi: 10.2514/6.2018-1437.

[3] Wilkie, W. K., Warren, J., Horta, L. G., Lyle, K. H., Juang, J.-N., Gibbs, S. C., Dowell, E., Guerrant, D. V., and Lawrence, D. A., "Recent Advances in Heliogyro Solar Sail Structural Dynamics, Stability, and Control Research," 2nd AIAA Spacecraft Structures Conference, Kissimmee, Florida, 2015. doi:10.2514/6.2015-0431

[4] Hashemi, M. R. M., Fikes, A. C., Gal-Katziri, M., Abiri, B., Bohn, F., Safaripour, A., Kelzenberg, M. D., Warmann, E. L., Espinet, P., Vaidya, N., Gdoutos, E. E., Leclerc, C., Royer, F., Pellegrino, S., Atwater, H. A., and Hajimiri, A., "A flexible phased array system with low areal mass density," Nature Electronics, Vol. 2, 2019, pp. 195-205. doi 10.1038/s41928-019-0247-9

[5] Arya, M., Lee, N., and Pellegrino, S., "Ultralight Structures for Space Solar Power Satellites," 3rd AIAA Spacecraft Structures Conference, San Diego, California, 2016. doi $10.2514 / 6.2016-1950$

[6] Gdoutos, E., Truong, A., Pedivellano, A., Royer, F., and Pellegrino, S., "Ultralight Deployable Space Structure Prototype," AIAA Scitech 2020 Forum, Orlando, Florida, 2020. doi 10.2514/6.2020-0692

[7] Armanini, C., Dal Corso, F., Misseroni, D., and Bigoni, D., "From the elastica compass to the elastica catapult: an essay on the mechanics of soft robot arm," Proceedings of the Royal Society A: Mathematical, Physical and Engineering Sciences, Vol. 473, No. 2198, 2017. doi $10.1098 /$ rspa.2016.0870

[8] Mobrem, M., and Adams, D. S., "Deployment Analysis of the Lenticular Jointed Antennas Onboard the Mars Express Spacecraft," Journal of Spacecraft and Rockets, Vol. 46, No. 2, 2009, pp. 394-402. doi $10.2514 / 1.36890$ 
[9] Mallikarachchi, H. M. Y. C., and Pellegrino, S., "Deployment Dynamics of Ultrathin Composite Booms with Tape-Spring Hinges,” Journal of Spacecraft and Rockets, Vol. 51, No. 2, 2014, pp. 604-613. doi 10.2514/1.A32401

[10] Pedivellano, A., Gdoutos, E., and Pellegrino, S., "Sequentially Controlled Dynamic Deployment of Ultra-Thin Shell Structures," AIAA Scitech 2020 Forum, Orlando, Florida, 2020. doi:10.2514/6.2020-0690

[11] Seffen, K. A., and Pellegrino, S., "Deployment dynamics of tape springs," Proceedings of the Royal Society A: Mathematical, Physical and Engineering Sciences, Vol. 455, No. 1983, 1999, pp. 1003-1048. doi 10.1098/rspa.1999.0347.

[12] Ryckelynck, D., "A priori hyperreduction method: an adaptive approach,” Journal of Computational Physics, Vol. 202, No. 1, 2005, pp. 346-366. doi $10.1016 /$ j.jcp.2004.07.015

[13] Kerschen, G., Golinval, J.-C., Vakakis, A. F., and Bergman, L. A., “The Method of Proper Orthogonal Decomposition for Dynamical Characterization and Order Reduction of Mechanical Systems: An Overview," Nonlinear Dynamics, Vol. 41, 2005, pp. 147-169. doi $10.1007 / \mathrm{s} 11071-005-2803-2$

[14] Farhat, C., Avery, P., Chapman, T., and Cortial, J., "Dimensional reduction of nonlinear finite element dynamic models with finite rotations and energy-based mesh sampling and weighting for computational efficiency," International Journal for Numerical Methods in Engineering, Vol. 98, No. 9, 2014, pp. 625-662. doi $10.1002 / \mathrm{nme} .4668$

[15] Farhat, C., Chapman, T., and Philip, A., "Structure-preserving, stability, and accuracy properties of the energy-conserving sampling and weighting method for the hyper reduction of nonlinear finite element dynamic models," International Journal for Numerical Methods in Engineering, Vol. 102, No. 5, 2015, pp. 1077-1110. doi $10.1002 / \mathrm{nme} .4820$

[16] Nash, M., "Nonlinear Structural Dynamics by Finite Element Modal Synthesis," Ph.D. thesis, Imperial College, London, 1977.

[17] Muravyov, A. A., and Rizzi, S. A., "Determination of nonlinear stiffness with application to random vibration of geometrically nonlinear structures," Computers \& Structures, Vol. 81, No. 15, 2003, pp. 1513-1523. doi 10.1016/S0045-7949(03)00145-7

[18] Kuether, R. J., Allen, M. S., and Hollkamp, J. J., "Modal Substructuring of Geometrically Nonlinear Finite-Element Models," AIAA Journal, Vol. 54, No. 2, 2016, pp. 691-702. doi $10.2514 / 1 . J 054036$

[19] Kuether, R. J., Allen, M. S., and Hollkamp, J. J., "Modal Substructuring of Geometrically Nonlinear Finite Element Models with Interface Reduction,” AIAA Journal, Vol. 55, No. 5, 2017, pp. 1695-1706. doi:10.2514/1.J055215

[20] Kerschen, G., Peeters, M., Vakakis, A. F., and Bergman, L. A., "Nonlinear normal modes, Part I: A useful framework for the structural dynamicist," Mechanical Systems and Signal Processing, Vol. 23, No. 1, 2009, pp. $170-194$. doi $10.1016 /$ j.ymssp.2008.04.002

[21] Idelsohn, S. R., and Cardona, A., "A reduction method for nonlinear structural dynamic analysis," Computer Methods in Applied Mechanics and Engineering, Vol. 49, No. 3, 1985, pp. 253-279. doi $10.1016 / 0045-7825(85) 90125-2$

[22] Idelsohn, S. R., and Cardona, A., "A load-dependent basis for reduced nonlinear structural dynamics," Computers \& Structures, Vol. 20, No. 1-3, 1985, pp. 203-210. doi $10.1016 / 0045-7949(85) 90069-0$

[23] Weeger, O., Wever, U., and Simeon, B., "On the use of modal derivatives for nonlinear model order reduction," International Journal for Numerical Methods in Engineering, Vol. 108, No. 13, 2016, pp. 1579-1602. doi 10.1002/nme.5267

[24] Wu, L., and Tiso, P., "Nonlinear model order reduction for flexible multibody dynamics: a modal derivatives approach," Multibody System Dynamics, Vol. 36, 2016, pp. 405-425. doi 10.1007/s11044-015-9476-5

[25] Wu, L., Tiso, P., Tatsis, K., Chatzi, E., and van Keulen, F., "A modal derivatives enhanced Rubin substructuring method for geometrically nonlinear multibody systems," Multibody System Dynamics, Vol. 45, 2019, pp. 57-85. doi 10.1007/s11044-01809644-2

[26] Noor, A. K., "On making large nonlinear problems small,” Computer Methods in Applied Mechanics and Engineering, Vol. 34, No. 1-3, 1982, pp. 955-985. doi $10.1016 / 0045-7825(82) 90096-2$

[27] Carlberg, K., Tuminaro, R., and Boggs, P., "Preserving Lagrangian Structure in Nonlinear Model Reduction with Application to Structural Dynamics," SIAM Journal on Scientific Computing, Vol. 37, No. 2, 2015, pp. B153-B184. doi $10.1137 / 140959602$

[28] Chaturantabut, S., and Sorensen, D. C., "Nonlinear Model Reduction via Discrete Empirical Interpolation," SIAM Journal on Scientific Computing, Vol. 32, No. 5, 2010, pp. 2737-2764. doi $10.1137 / 090766498$ 
[29] Everson, R., and Sirovich, L., "Karhunen-Loéve procedure for gappy data," Journal of the Optical Society of America A, Vol. 12, No. 8, 1995, pp. 1657-1664. doi 10.1364/JOSAA.12.001657

[30] Astrid, P., Weiland, S., Willcox, K., and Backx, T., "Missing Point Estimation in Models Described by Proper Orthogonal Decomposition," IEEE Transactions on Automatic Control, Vol. 53, No. 10, 2008, pp. 2237-2251. doi 10.1109/TAC.2008.2006102.

[31] Hernández, J. A., Caicedo, M. A., and Ferrer, A., "Dimensional hyper-reduction of nonlinear finite element models via empirical cubature," Computer Methods in Applied Mechanics and Engineering, Vol. 313, 2017, pp. 687-722. doi $10.1016 /$ j.cma.2016.10.022

[32] Jain, S., and Tiso, P., "Simulation-Free Hyper-Reduction for Geometrically Nonlinear Structural Dynamics: A Quadratic Manifold Lifting Approach,” Journal of Computational and Nonlinear Dynamics, Vol. 13, No. 7, 2018. doi 10.1115/1.4040021

[33] Sipp, D., Fosas de Pando, M., and Schmid, P. J., "Nonlinear model reduction: a comparison between POD-Galerkin and POD-DEIM methods," Computers \& Fluids, Vol. 208, 2020, p. 104628. doi 10.1016/j.compfluid.2020.104628.

[34] Cardona, A., and Géradin, M., “Modelling of superelements in mechanism analysis," International Journal for Numerical Methods in Engineering, Vol. 32, No. 8, 1991, pp. 1565-1593. doi $10.1002 / \mathrm{nme} .1620320805$

[35] Shabana, A. A., Dynamics of Multibody Systems, $4^{\text {th }}$ ed., Cambridge University Press, New York, 2013, Chaps. 5, 6, pp. 185-303. doi $10.1017 /$ CBO9781107337213

[36] Nikravesh, P. E., "Understanding Mean-Axis Conditions as Floating Reference Frames," Advances in Computational Multibody Systems, Vol. 2, Springer, Dordrecht, Netherlands, 2005, pp. 185-203. doi 10.1007/1-4020-3393-1.

[37] Zienkiewicz, O. C., Taylor, R. L., and Zhu, J. Z., The Finite Element Method: Its Basis and Fundamentals, 6 ${ }^{\text {th }}$ ed., Butterworth-Heinemann, Oxford, United Kingdom, 2006, Chap. 16, pp. 568-570.

[38] Simo, J. C., and Vu-Quoc, L., "The role of non-linear theories in transient dynamic analysis of flexible structures," Journal of Sound and Vibration, Vol. 119, No. 3, 1987, pp. 487-508. doi $10.1016 / 0022-460 X(87) 90410-X$

[39] Carpenter, N., Belytschko, T., and Stolarski, H., "Locking and shear scaling factors in $\mathrm{C}^{0}$ bending elements," Computers \& Structures, Vol. 22, No. 1, 1986, pp. 39-52. doi 10.1016/0045-7949(86)90083-0

[40] Thomas, O., Sénéchal, A., and Deü, J.-F., "Hardening/softening behavior and reduced order modeling of nonlinear vibrations of rotating cantilever beams," Nonlinear Dynamics, Vol. 86, 2016, pp. 1293-1318. doi 10.1007/s11071-016-2965-0

[41] Crisfield, M. A., "A fast incremental/iterative solution procedure that handles "snap-through"," Computers \& Structures, Vol. 13, No. 1-3, 1981, pp. 55-62. doi 10.1016/0045-7949(81)90108-5

[42] Arnold, M., and Brüls, O., "Convergence of the generalized- $\alpha$ scheme for constrained mechanical systems," Multibody System Dynamics, Vol. 18, 2007, pp. 185-202. doi $10.1007 /$ s11044-007-9084-0

[43] Chung, J., and Hulbert, G. M., “A Time Integration Algorithm for Structural Dynamics With Improved Numerical Dissipation: The Generalized- $\alpha$ Method," Journal of Applied Mechanics, Vol. 60, No. 2, 1993, pp. 371-375. doi $10.1115 / 1.2900803$

[44] Cardona, A., and Géradin, M., "Time integration of the equations of motion in mechanism analysis," Computers \& Structures, Vol. 33, No. 3, 1989, pp. 801-820. doi 10.1016/0045-7949(89)90255-1

[45] Bottasso, C. L., Dopico, D., and Trainelli, L., "On the optimal scaling of index three DAEs in multibody dynamics," Multibody System Dynamics, Vol. 19, 2008, pp. 3-20. doi 10.1007/s11044-007-9051-9. 